\title{
Position-specific information in social networks: Are you connected?
}

\author{
Michael McBride* \\ Department of Economics, University of California, 3151 Social Science Plaza, Irvine, CA, 92697-5100, United States
}

\section{A R T I C L E I N F O}

\section{Article history:}

Received 31 July 2007

Received in revised form

26 February 2008

Accepted 9 May 2008

Available online 22 May 2008

\section{JEL classification:}

A14

C72

D20

D80

Keywords:

Limited horizons

Observation

Communication

Connections

\begin{abstract}
A B S T R A C T
Individuals in social networks often imperfectly monitor others' network relationships and have incomplete information about the value of forming new relationships. This paper introduces the Generalized Conjectural Equilibrium (GCE) concept for such settings and completely characterizes the set of GCE networks when players observe only local parts of the network. Incomplete information and imperfect monitoring generate different types of inefficiency. These inefficiencies increase in number and scope as network observation becomes more localized. These results suggest that actual social networks will be structured inefficiently in general.
\end{abstract}

(c) 2008 Elsevier B.V. All rights reserved.

\section{Introduction}

Social networks underlie many social activities, from the trade of goods and services in noncentralized markets, to the spread of information about job openings. Yet, all of these social networks have a common feature that has been largely ignored in theoretical work: individuals initiate and maintain social ties with very incorrect beliefs about the structure of the network. ${ }^{1}$ Sociologists acknowledge that "actors optimize based on local information only" [Stokman and Doreian (1997), 246, their emphasis] even though researchers do not incorporate the idea systematically.

\footnotetext{
* Tel.: +1 9498247417.

E-mail address: mcbride@uci.edu.

${ }^{1}$ This feature has been empirically studied in various sociological research. For example, see Laumann (1969), Kumbasar et al. (1994), Bondonio (1998), and Casciaro (1998).
} 
Economists have generally ignored the fact and assumed that individuals have full and global information. $^{2}$

This paper examines the role local information plays in network formation by focusing on two distinct types of informational limitations. First, an individual usually imperfectly monitors who is connected to whom in the network. Second, she has incomplete information about the value of certain connections. Will such limited information lead to the persistence of networks that differ from those that would arise under full information? If so, why, and are the differences economically meaningful? Does imperfect monitoring affect network formation differently than incomplete information? This paper examines these questions using a systematic game theoretic approach. I use as a starting point a model of network formation first introduced by Bala and Goyal (2000) and extended by Galeotti et al. (2006) and McBride (2006a). Each individual has a "fact" that can be communicated through the network, and each player derives utility from another's fact only if she learns it through the network.

I introduce two formal tools in my analysis: the Generalized Conjectural Equilibrium (GCE) concept and $x / y$-link observation. The GCE concept relaxes the restriction that each individual has correct beliefs in equilibrium and instead allows an individual's equilibrium beliefs to be incorrect, as observed in the real world, as long as they are consistent with the her limited information. According to $x / y$-link observation, an individual monitors all social ties that are within $x$ ties away and observes the types of all individuals that are within $y$ ties from her in the network. The main result is a complete characterization of GCE networks under $x / y$-link observation. As position-specific information becomes more limited (as $x$ and $y$ decrease), the number of inefficient equilibria increases. Intuitively, observing less of one's network prevents one from identifying, and thus eliminating, network inefficiencies. A second finding is that the two informational limitations - imperfect monitoring and incomplete information - have fundamentally different equilibrium implications. The ability to monitor actions generally allows individuals to remove over-connections but does not compel individuals to overcome under-connections because unseen opportunities will be missed. Knowledge of types allows individuals to identify under-connections, but does not allow individuals to identify over-connections. Efficiency may require both types of information to be present.

Overall, this paper provides an equilibrium-based theoretical study of the empirical finding that individuals maintain incorrect perceptions of their networks. It is the first game theoretic study of both imperfect monitoring and incomplete information in social networks. McBride (2006a), which considers imperfect monitoring, is the first paper to relax the full information assumption and use a non-Nash Equilibrium concept to study networks. This paper differs in also considering incomplete information and by introducing the issue of how different types of informational limitations lead to different types of network inefficiencies. McBride (2006b) adapts the GCE concept introduced herein to a mutual consent network formation setting. This body of work shows how persistently incorrect beliefs generate a variety of network outcomes. ${ }^{3}$

\section{Model, equilibrium, and network observation}

\subsection{Players, strategies, and payoffs}

Each player $i \in N=\{1, \ldots, n\}$ knows a "fact" that is valued by all other players. The fact might be investment information, valued news, insights into a new productive technology, etc. Let $v_{i}$ denote $i$ 's type, which captures the value of $i$ 's fact to others. Let $v=\left(v_{1}, \ldots, v_{n}\right)$ be the profile of values and $V$ be the set of possible profiles.

Player $i$ learns $j$ 's fact only through a direct bilateral tie with $j$ or indirectly through a path of other players' direct ties. The tie exists if one or both players initiate a communication link, where initiating

\footnotetext{
2 See Dutta and Jackson (2003) for a collection of many of the important contributions to this field.

${ }^{3}$ Although McBride (2006b) was published before this paper, this paper was actually written first. McBride (2006b) cites and builds upon this paper.
} 

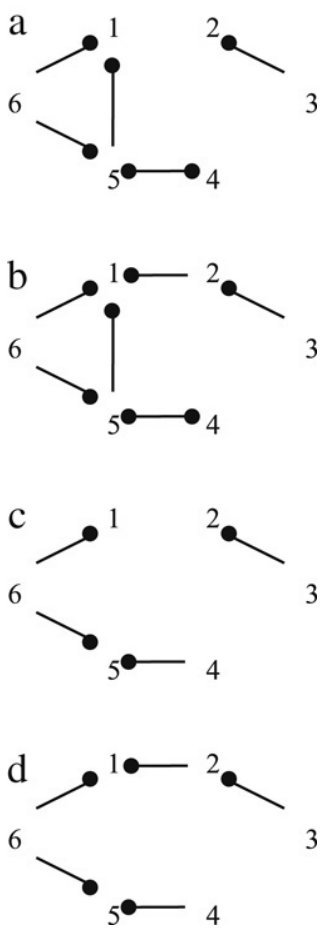

Fig. 1.

a link costs $c<\infty$ to the initiator. The cost captures the time, effort, or money invested to form and maintain the link. Each $i$ must choose with whom to initiate links, which constitutes a vector $s_{i}=\left(s_{i 1}, \ldots, s_{i i-1}, s_{i i+1}, \ldots, s_{i n}\right)$, where $s_{i j}=1$ signifies that $i$ initiates a link to $j$, and $s_{i j}=0$ signifies that $i$ does not initiate a link to $j$. Denote $S_{i}$ the set of $i$ 's possible link decisions, and let $S=S_{1} \times \cdots \times S_{n}$, where $s=\left(s_{1}, \ldots, s_{n}\right) \in S$ is one possible profile of link choices that constitute a network structure or graph. A node in the graph represents an individual player, and the bilateral ties between nodes represent communication links.

There is a path between $i$ and $j$ if they are directly linked, $\max \left\{s_{i j}, s_{j i}\right\}=1$, or if they are indirectly linked such that there exist players $j_{1}, \ldots, j_{m}$ distinct from each other and from $i$ and $j$ such that $\max \left\{s_{i j_{1}}, s_{j_{1} i}\right\}=\max \left\{s_{j_{1} j_{2}}, s_{j_{2} j_{1}}\right\}=\cdots=\max \left\{s_{j_{m j}}, s_{j j_{m}}\right\}=1$. Define a network component to be a subset of players such that there exists a path between any two players in the subset, and there is no path between a player in the subset and a player not in the subset. Given structure $s$, denote $N_{i} \subseteq N$ to be $i$ 's component. Let $I_{i}=\left\{j \in N \mid s_{i j=1}\right\}$ be the set of $i$ 's link initiations and $\left|I_{i}\right|$ be its cardinality.

Each player has utility function $u_{i}\left(s_{i}, s_{-i} \mid v\right)=\sum_{j \in N_{i}} v_{j}-\left|I_{i}\right| c$. The value of $j$ 's fact to $i$ does not depend on how many links away $j$ is in $i$ 's component so long as she is in $i$ 's component (see Section 5 for a brief consideration of flow decay).

Fig. 1(a) illustrates one possible $s$. Following convention for this model, a dot indicates which node of the tie is the link initiator. For player $1, N_{1}=\{1,4,5,6\}, I_{1}=\{5,6\}$, and $\left|I_{i}\right|=2$. Note that players in the same component can have different utilities when they make different numbers of link initiations. An important feature of a network is its connectivity. A network like Fig. 1(b) in which all players are in one component is called connected. Fig. 1(a) is disconnected because $N_{i} \subset N$ for all $i$. A special case of a disconnected network is the empty network in which each player is isolated, i.e., $N_{i}=\{i\}$ for all $i$. Another important feature is whether or not a network has redundant link initiations called cycles. Fig. 1(c) is identical to Fig. 1(a) but with two redundant link initiations removed: $s_{15}=s_{45}=0$. A network without cycles is called minimal. Fig. 1(d) depicts a minimally 
connected network, which is of interest because any efficient (maximized sum of utilities) network that is non-empty must be minimally connected. ${ }^{4}$

This model captures many features of actual social networks. ${ }^{5}$ Valuable information is often communicated through networks that arise from the uncoordinated decisions of individuals to form or sever ties. These networks span large distances, so it is likely that an individual will not observe all others' ties (imperfect monitoring) nor the specific value of another person's information (incomplete information). Thus, this model is rich enough to capture these observational details, but also simple enough to conduct formal analysis.

\subsection{Generalized conjectural equilibrium}

The Nash Equilibrium (NE) concept and its refinements restrict players to commonly know $v$ and $s$ in equilibrium. Other variations on the NE concept also make a similar restriction. For example, the Bayesian NE concept for games of incomplete information assumes that players commonly know the prior probability distribution over types. Instead of forcing this convergence in beliefs, I generalize the Conjectural Equilibrium (CE) concept, which was designed for games with imperfect monitoring to also consider incomplete information. I first define a game of incomplete information and imperfect monitoring.

Definition 1. A game of incomplete information and imperfect monitoring is a combination

$$
\left\langle N, \Theta, A, \Pi,\left(u_{i}\right)_{i \in N},\left(m_{i}\right)_{i \in N}\right\rangle,
$$

where: $N$ is a set of players; $\Theta$ is a set of states; $A_{i}$ is the set of actions for $i \in N$ and $A=\times_{i \in N} A_{i} ; \Pi_{i}$ is $i$ 's set of probability distributions over $A \times \Theta$ and $\Pi=\times_{i \in N} \Pi_{i \in N} ; u_{i}: A \times \Theta \rightarrow R$ is $i$ 's utility function; and $m_{i}: A \times \Theta \rightarrow M_{i}$ is $i$ 's signal or message function with message space $M_{i}$.

The inclusion of signal functions distinguishes this game from a standard game of incomplete information. The signal function is used in games of imperfect monitoring to formalize an individual's monitoring of others' actions. Here, the signal function also captures the subset of others' types monitored.

Definition 2. Fix $\theta \in \Theta$. A Generalized Conjectural Equilibrium (GCE) of the game $\left\langle N, \Theta, A, \Pi,\left(u_{i}\right)_{i \in N}\right.$, $\left.\left(m_{i}\right)_{i \in N}\right\rangle$ is a profile of actions and beliefs $\left(a_{i}^{*}, \pi_{i}^{*}\right)_{i \in N} \in A \times \Pi$ such that for each $i$ :

(i) $\sum_{\left(a_{-i}^{\prime}, \theta^{\prime}\right) \in\left\{A_{-i} \times \Theta\right\}} \pi_{i}^{*}\left(a_{i}^{*}, a_{-i}^{\prime}, \theta^{\prime}\right) u_{i}\left(a_{i}^{*} \mid a_{-i}^{\prime}, \theta^{\prime}\right) \geq \sum_{\left(a_{-i}^{\prime}, \theta^{\prime}\right) \in\left\{A_{-i} \times \Theta\right\}} \pi_{i}^{*}\left(a_{i}^{\prime \prime}, a_{-i}^{\prime}, \theta^{\prime}\right) u_{i}\left(a_{i}^{\prime \prime} \mid a_{-i}^{\prime}\right.$, $\left.\theta^{\prime}\right) \forall a_{i}^{\prime \prime} \in A_{i}$,

(ii) For any $\left(a^{\prime}, \theta^{\prime}\right) \in A \times \Theta$ s.t. $\pi_{i}^{*}\left(a^{\prime}, \theta^{\prime}\right)>0$, it must be that $m_{i}\left(a^{\prime}, \theta^{\prime}\right)=m_{i}\left(a^{*}, \theta\right)$.

(iii) For any $\left(a^{\prime}, \theta^{\prime}\right) \in A \times \Theta$ s.t. $u_{i}\left(a^{\prime} \mid \theta^{\prime}\right) \neq u_{i}\left(a^{*} \mid \theta\right)$, it must be that $\pi_{i}^{*}\left(a^{\prime}, \theta^{\prime}\right)=0$.

Condition (i) states that in equilibrium each $i$ 's action $a_{i}^{*}$ must be a best response given her conjectured beliefs $\pi_{i}^{*}$. Condition (ii) places a restriction on each $i$ 's beliefs: for any state of the world $\left(a^{\prime}, \theta^{\prime}\right)$ that $i$ assigns non-zero probability, it must be true that the message $i$ receives in that state equals the message $i$ receives in the true state of the world $\left(a^{*}, \theta\right)$. In other words, a player's beliefs must not contradict her message. Condition (iii) restricts beliefs so that $i$ cannot assign non-zero probability to any state of the world that her knowledge of her own utility tells her cannot be the true state of the world. This condition follows from the assumption mentioned above that signals reveal at least as much information as the payoff.

A CE is a GCE of a game with complete information (e.g., $\Theta=\{\theta\}$ or $\theta$ publicly observed). As a static concept, GCE, like NE, does not describe how equilibrium beliefs form. It only holds that a particular profile of beliefs and actions constitutes an equilibrium if the GCE conditions are met. We can, however, motivate the GCE concept similar to how the CE and Self-Confirming Equilibrium

\footnotetext{
${ }^{4}$ For a formal proposition and proof of this claim, see the technical appendix on the author's web site http://www.econ.uci.edu/ mcbride/.

${ }^{5}$ See Wasserman and Faust (1994) for a general discussion of social network properties.
} 
concepts have been motivated (Gilli, 1999; Fudenburg and Levine, 1993). If a static game is repeated over time and players receive only limited information about other players' types and strategies as the game progresses, then incorrect beliefs can persist over time, and players might not ever receive information to contradict those incorrect beliefs. ${ }^{6}$ The primary disadvantage of the GCE concept is that it places so few restrictions on equilibrium beliefs that the set of equilibria can often be very large. However, the GCE conditions can be viewed as the minimum necessary conditions for an equilibrium. This also guards against making restrictions difficult to justify in the network setting with limited observation. The underlying question concerns what should $i$ assume about the actions and types of individuals she does not observe. Restricting players to have common knowledge of $F_{v}$, for example, as assumed in a Bayesian Equilibrium, is difficult to justify when each player observes a different part of the network.

\subsection{Network observation}

To apply the GCE concept to the network formation game, we must specify players' signal functions. I here propose the notion of $x / y$-link observation, which mimics the incomplete information and imperfect monitoring present in actual social networks. ${ }^{7}$ Given network $s$, let $d(i, j \mid s)$ be the distance of the shortest path between $i$ and $j$, where $d(i, i \mid s)=0$ and $d(i, j \mid s)=\infty$ if $j \notin N_{i}$. Let $N_{i}^{x / y}(s)$ be the set of links that are observed by $i$ given $s$ under $x / y$-link observation: $N_{i}^{x / y}=\left\{s_{j} \mid d(i, j) \leq x-1\right\} \cup$ $\left\{s_{k j} \mid d(i, k)=x\right.$ and $\left.d(i, j)=x-1\right\}$.

Definition 3. Given $(s, v)$, signal function $m_{i}^{x / y}(s, v)$ exhibits $x / y$-link observation if the signal contains (i) $N_{i}^{x / y}(s)$ and (ii) $\left\{v_{j} \mid d(i, j) \leq y\right\}$.

Assume for now (relaxed later) that $x \geq 1$ and $0 \leq y \leq x$. These are natural restrictions: an individual knows with whom she has direct links and should only be able to observe another's type if she observes that player in the network $(y \leq x)$, and observing another's links does not imply observing her type ( $y$ can be strictly less than $x$ ). In Fig. 2(a), the dashed line encloses the links observed by player 1 when $x=1$, and the dashed circles show the players whose types are observed when $y=0$. 1 observes her link initiation to 6,5 's link initiation to her, her own type, and nothing else. As $x$ or $y$ or both increase, $i$ observes weakly more of her network. Fig. 2(b) depicts 1's signal with $x=2$ and $y=2$, where 1 now observes two more links and three more players' types. Individual $i$ never observes any player not in her component if $x$ and $y$ are finite. Finally, note that $x / y$-link observation mimics to some extent what individuals observe in actual social networks. Individuals gain their information about the network through their own network interactions, and they are more likely to observe the parts of the network closer to themselves. ${ }^{8}$

\section{Characterization of network equilibria}

To completely characterize the set of network equilibria requires additional notation. For $j \in I_{i}$, let $P_{i j}=\{k \in N \mid$ there is a path from $i$ to $k$ through $j\}$, and let $P_{i j}^{x / y}=\{k \in N \mid$ there is a path from $i$ to $k$ through $j$ and $d(i, k \mid s) \leq x$. In words, $P_{i j}$ is the set of all players on the path from $i$ to $j$, while $P_{i}^{x / y}$ is the set of all players on the path that are observed in the signal $m_{i}^{x / y}(s, v)$. Note that $k$ is in $P_{i j}$ but not in $P_{i j}^{x / y}$ if the path from $i$ through $j$ to $k$ exists but is not observed.

\footnotetext{
6 See Battigalli et al. (1992) and Gilli (1999) for extended discussions of the Conjectural Equilibrium (CE) concept in games with imperfect monitoring. While I generalize the CE concept, it has also been further refined. Fudenburg and Levine's (1993) Self-confirming Equilibrium is a CE in which i's signal contains the strategies that all others play at all information sets on the equilibrium path. Rubinstein and Wolinsky's (1994) Rationalizable Conjectural Equilibrium further assumes common knowledge of rationality.

7 This $x / y$-link observation concept generalizes the $x$-link observation concept introduced by McBride (2006a).

8 See Friedkin (1983), Kumbasar et al. (1994), Bondonio (1998), and Casciaro (1998).
} 
a

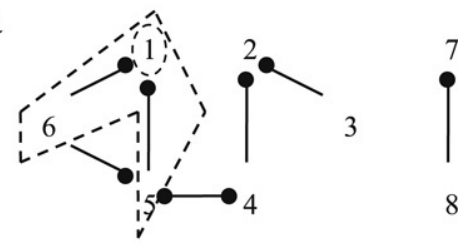

b
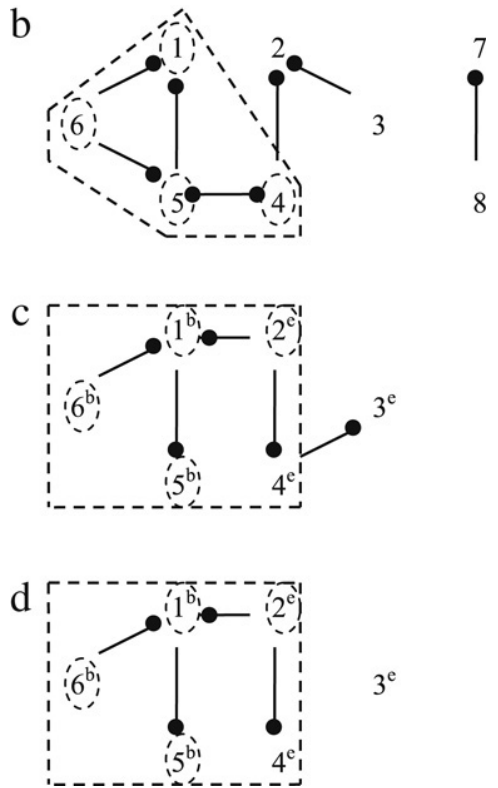

Fig. 2.

Let $v_{i j+}(s, v)$ be the actual marginal value of $i$ 's link to $j$ :

$$
v_{i j+}(s, v)= \begin{cases}0, & \text { if } j \text { and } i \text { are in a cycle } \\ \sum_{k \in P_{i j}} v_{k}, & \text { otherwise. }\end{cases}
$$

Let $v_{i j+}^{x / y}(s, v)$ be the observed marginal value of $i$ 's link with $j$ :

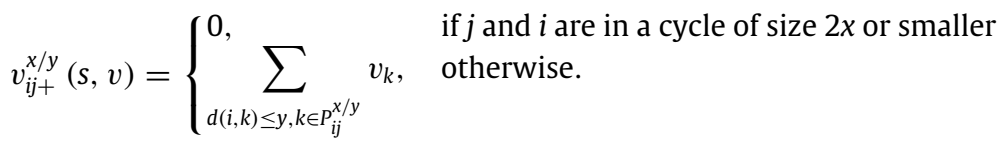

Say that component $N_{i}$ has a "low-valued, link-receiving subcomponent" (LLS) if there exists a $j \in N_{i}$ with $s_{i j}=1$ such that setting $s_{i j}=0$ partitions $N_{i}$ into two separate components $N_{i}^{\prime}$ and $N_{j}^{\prime}$ and $0<v_{i j+}(s, v)<c$. Intuitively, any $i$ would remove a link to $j$ if $i$ knows $j$ is in a LLS because the link to $j$ provides benefits less than cost $c$. Let $L L S_{i}^{x / y} \equiv\left\{j \in I_{i} \mid v_{i j+}^{x / y}(s, v)<c\right\}$ be the players to whom $i$ initiates links that could be a LLS given her observation. Notice that $j \in L L S_{i}^{x / y}$ does not imply that $j$ is indeed part of a LLS; it just implies that $j$ might be part of a LLS.

Let $\widetilde{v}_{i}^{x / y}(s, v) \equiv u_{i}(s)-\sum_{j \in N \text { s.t. } d(i, j \mid s) \leq y} v_{j}$ be $i$ 's utility that is received but unaccounted for given her observation. Finally, define $\widetilde{v}_{i j+}^{x / y}\left(s^{\prime}, v^{\prime}\right)=v_{i j+}\left(s^{\prime}, v^{\prime}\right)-v_{i j+}^{x / y}(s, v)$. Note that $v_{i j+}^{x / y}(s, v)$ is known 
by $i$, but because $i$ might assign non-zero probability to some state $\left(s^{\prime}, v^{\prime}\right), \widetilde{v}_{i j+}^{x / y}\left(s^{\prime}, v^{\prime}\right)$ captures what $i$ would see as that part of the marginal value of the link with $j$ that is unobserved in state $\left(s^{\prime}, v^{\prime}\right)$. If the link is redundant in state $\left(s^{\prime}, v^{\prime}\right)$, then $\widetilde{v}_{i j+}^{x / y}\left(s^{\prime}, v^{\prime}\right)=-v_{i j+}^{x / y}(s, v)$.

To visualize these definitions, see Fig. 2(c), where the " $\mathrm{b}$ " next to 1 means $v_{1}=b$, and so on, assuming $e<c<b$. In that network, $N_{1}^{2 / 1}(s)$ would be depicted as Fig. $2(\mathrm{~d})$, because 1 does not observe the link between 3 and 4 . We have $P_{12}(s, v)=\{2,3,4\}, P_{12}^{2 / 1}(s, v)=\{2,4\}$, and $d(1,3 \mid s)=3$. The marginal value of 1 's link to 2 is $v_{12+}(s, v)=3 e$, and the observed marginal value is $v_{12}^{2 / 1}(s, v)=e$. The set of 1's possible LLSs is $L L S_{1}^{2 / 1}=\{2\}$ because $v_{12}^{2 / 1}(s, v)=e<c<$ $v_{16}^{2 / 1}(s, v)=b$. 1's total unaccounted for utility is $\widetilde{v}_{1}^{2 / 1}(s, v)=(3 b+3 e-2 c)-(3 b-e-2 c)=2 e$.

We can now state and prove the main proposition of the paper, where $E_{x / y}(v)$ is the set of equilibrium networks that can be sustained as equilibria given type profile $v$ under $x / y$-link observation.

Proposition 1. Fix $v$. $E_{x / y}(v)$ consists of all $s^{*}$ such that

(a) $s^{*}$ has no cycles of size less than or equal to $2 x$;

(b) $\widetilde{v}_{i}^{x / y}\left(s^{*}, v\right) \geq \sum_{j \in L L S_{i}^{x / y}}\left(c-v_{i j+}^{x / y}\left(s^{*}, v\right)\right)$ for any $i$ with $\left|L L S_{i}^{x / y}\right| \geq 1$;

(c) for each $i$ with $\left|L L S_{i}^{x / y}\right| \geq 1$, (i) if $x=y$ then $N_{i}^{x / y} \subset N$, and (ii) if $x>y$, then for each $j \in L L S_{i}^{x / y}$, there is a $k \in N$ on a path through $j$ s.t. $d\left(i, k \mid s^{*}\right)=y+1$.

Proof. Necessity. (a) Suppose $s^{*}$ has a cycle of size $z \leq 2 x$. Consider $i$ and $j$ in the cycle with $s_{i j}=1$. It must be true that $m_{i}^{x / y}\left(s^{*}, v\right)$ reveals that cycle to $i$, and $\pi_{i}^{*}$ must assign probability 0 to any $s$ without that cycle. Given such $\pi_{i}^{*}$, it must be true that $i$ believes she is strictly better off by setting $s_{i j}=0$. But this contracts GCE condition (i).

(b) According to GCE condition (i), to not remove any link to any $j \in L L S_{i}^{x / y}$, it is necessary that there is enough $\widetilde{v}_{i}^{x / y}(\cdot)$ to make the link with any $j \in L L S_{i}^{x / y}$ not be an LLS:

$$
\sum_{\left(s^{\prime}, v^{\prime}\right) \in(S \times V)} \pi^{*}\left(s^{\prime}, v^{\prime}\right)\left(\widetilde{v}_{i j+}^{x / y}\left(s^{\prime}, v^{\prime}\right)+v_{i j+}^{x / y}(s, v)\right) \geq c .
$$

Because $v_{i j+}^{x / y}(s, v)$ must be the same in any $\left(s^{\prime}, v^{\prime}\right)$ assigned non-zero probability, this becomes

$$
\sum_{\left(s^{\prime}, v^{\prime}\right) \in(S \times V)} \pi^{*}\left(s^{\prime}, v^{\prime}\right) \widetilde{v}_{i j+}^{x / y}\left(s^{\prime}, v^{\prime}\right) \geq c-v_{i j+}^{x / y}(s, v) .
$$

This inequality must hold for all $j \in L L S_{i}^{x / y}$, so we can sum across those $j$ 's to get

$$
\sum_{j \in L L S_{i}^{x / y}} \sum_{\left(s^{\prime}, v^{\prime}\right) \in(S, V)} \pi_{i}^{*}\left(s^{\prime}, v^{\prime}\right) \widetilde{v}_{i j+}^{x / y}\left(s^{\prime}, v^{\prime}\right) \geq \sum_{j \in L L S_{i}^{x / y}}\left(c-v_{i j+}^{x / y}(s, v)\right),
$$

and because the left hand side cannot exceed the total amount of unaccounted for utility $\widetilde{v}_{i}^{x / y}\left(s^{*}, v\right)$, it follows that

$$
\widetilde{v}_{i}^{x / y}\left(s^{*}, v\right) \geq \sum_{j \in L L S_{i}^{x / y}}\left(c-v_{i j+}^{x / y}(s, v)\right) .
$$

(c-i) Suppose the contrary, i.e., for $i$ with $\left|L L S_{i}^{x / y}\right| \geq 1$ and $x=y$, assume that $N_{i}^{x / y}=N$. Consider $j \in L L S_{i}^{x / y}$, and let $k$ be the player furthest from $i$ in $P_{i j}^{x / y}$. Any $\left(s^{\prime}, v^{\prime}\right)$ assigned non-zero probability by $\pi_{i}^{*}$ either has no player $k^{\prime}$ with $d\left(i, k^{\prime}\right)=y+1$ or has a player $k^{\prime}$ with $d\left(i, k^{\prime}\right)=y+1$. In the first case, $i$ is strictly better off removing the link with $j$ because the link is an LLS in $\left(s^{\prime}, v^{\prime}\right)$. In the second case, because $k^{\prime} \in N_{i}$, the link with $j$ must be a cycle, so removing the link makes $i$ strictly better off. 
Because $\pi_{i}^{*}$ is a weighted average, it follows that $i$ believes she will be strictly better off removing the link. But this contradicts GCE condition (i).

(c-ii) Suppose the contrary, i.e., for $i$ with $\left|L L S_{i}^{x / y}\right| \geq 1$ and $x>y$, there is a $j \in L L S_{i}^{x / y}$ without a $k \in N$ s.t. $d\left(i, k \mid s^{*}\right)=y+1$. Because $x>y, m_{i}^{x / y}\left(s^{*}, v\right)$ must reveal to $i$ that there is no such $k$. By GCE condition (ii), $\pi_{i}^{*}$ must assign probability 0 to any state with such a $k$. But with these $\pi_{i}^{*}, i$ believes with probability 1 that the link to $j$ is a LLS and that she is strictly better off removing her link to $j$. This contradicts GCE condition (i).

Sufficiency for $i$ with $\left|L L S_{i}^{x / y}\right| \geq 1$ and $x=y$. Suppose $x=y$, and consider $s^{*}$ and $v$ that satisfy (a), (b), and (c-i) with $\left|L L S_{i}^{x / y}\right| \geq 1$ for some $i$. Consider $i$ with $L L S_{i}^{x / y}=\left\{j_{1}, \ldots, j_{z}\right\}, z \geq 1$, and consider $k \notin N_{i}^{x / y}\left(s^{*}\right)$. Construct $z$ different states $s^{1}, \ldots, s^{z}$ in the following manner: each has subnetwork $N_{i}^{x / y}\left(s^{*}\right)$; state $s^{1}$ has $k$ initiate a link to player $l_{1}$ who is $x$ links away from $i$ through $j_{1}$, state $s^{2}$ has $k$ initiate to $l_{2}$ who is $x$ links away from $i$ through $j_{2}$, and so on; and any $j \notin\left\{N_{i}^{x / y} \cup\{k\}\right\}$ is isolated. Let $v^{\prime}$ have $v_{j}^{\prime}=v_{j}$ as observed for all $j \in N_{i}^{x / y}, v_{k}^{\prime}=\widetilde{v}_{i}^{x / y}\left(s^{*}, v\right)$, and set $v_{j}^{\prime}=\bar{v}<c$ for all $j \notin\left\{N_{i}^{x / y}\left(s^{*}\right) \cup\{k\}\right\}$. Notice that (b) is satisfied. Finally, set

$$
\pi_{i}^{*}\left(s^{t}, v^{\prime}\right)=\frac{c-v_{i j_{t}}^{x / y}}{\sum_{j \in L L S_{i}^{x / y}}\left(c-v_{i j t}^{x / y}\right)}
$$

for $t=1, \ldots, z$. As constructed, $\sum_{\left(s^{\prime}, v^{\prime}\right) \in(S \times V)} \pi_{i}^{*}\left(s^{\prime}, v^{\prime}\right)=1$, and GCE conditions (ii) and (iii) are met for $i$.

To show that condition (i) is met, notice that, given $\pi_{i}^{*}$, the expected cost of removing the link with $j_{t}, t=1, \ldots, z$, must exceed the expected benefit of removing the link:

$$
\frac{c-v_{i j_{t}}^{x / y}}{\sum_{j \in L L S_{i}^{x / y}}\left(c-v_{i j t}^{x / y}\right)} \widetilde{v}_{i}+v_{i j t}^{x / y} \geq c .
$$

With (b) satisfied, it must be true that

$$
\frac{c-v_{i j_{t}}^{x / y}}{\sum_{j \in L L S_{i}^{x / y}}\left(c-v_{i j_{t}}^{x / y}\right)} \widetilde{v}_{i}+v_{i j_{t}}^{x / y} \geq \frac{c-v_{i j_{t}}^{x / y}}{\widetilde{v}_{i}} \widetilde{v}_{i}+v_{i j_{t}}^{x / y}=c-v_{i j_{t}}^{x / y}+v_{i j_{t}}^{x / y}=c,
$$

so keeping the link is a best response. Adding links is not a best response because, according to $\pi_{i}^{*}$, all $j \notin N_{i}^{x / y} \cup\{k\}$ are isolated with $v_{j}<c$ as constructed. Thus, GCE condition (i) holds for $i$.

Sufficiency for $i$ with $\left|L L S_{i}^{x / y}\right| \geq 1$ and $x>y$. Suppose $x>y$, and consider $s^{*}$ and $v$ that satisfy (a), (b), and (c-ii) with $\left|L L S_{i}^{x / y}\right| \geq 1$ for some $i$. Consider $i$ with $L L S_{i}^{x / y}=\left\{j_{1}, \ldots, j_{z}\right\}, z \geq 1$. Let state $s^{\prime}$ have $N_{i}^{x / y}\left(s^{*}\right)$ as does $s^{*}$, but make any $j \notin N_{i}^{x / y}(s)$ isolated. By (a), $s^{\prime}$ is minimal. Set $v_{j}^{\prime}=v_{j}$ for values observed given $y$; set $v_{k_{1}}^{\prime}=c-v_{i j_{1}+}\left(s^{*}, v\right)$ for $k_{1}$ from (c-ii), set $v_{k_{2}}^{\prime}=c-v_{i j_{2}+}\left(s^{*}, v\right)$ for $k_{2}$, and so on for $k_{3}, \ldots, k_{z-1}$; set $v_{k_{z}}^{\prime}=\widetilde{v}_{i}^{x / y}-\sum_{t=1}^{z-1} v_{k_{t}}^{\prime}$; and set $v_{j}^{\prime}=0$ for all others. Notice that (b) is satisfied and that $i$ 's expected payoff under $\left(s^{\prime}, v^{\prime}\right)$ equals that under the true state $\left(s^{*}, v\right)$. Finally, set $\pi_{i}^{*}\left(s^{\prime}, v^{\prime}\right)=1$. As constructed, $\pi_{i}^{*}$ meets GCE conditions (ii) and (iii) for $i$. Moreover, given $\pi_{i}^{*}$, removing any link yields a weak decrease in expected payoff, while adding a link yields a strict decrease in expected payoff. Thus, GCE condition (i) is also met for $i$. 
Sufficiency with $\left|L L S_{i}^{x / y}\right|=0$ for $i$. Suppose $s^{*}$ and $v$ that satisfy (a) and (b), and consider $i$ with $\left|L L S_{i}^{x / y}\right|=0$. Set $\pi_{i}^{*}\left(s^{\prime}, v\right)=1$ where $s^{\prime}$ equals $s^{*}$ except that for any cycle of size $2 x+1$ or larger in $s^{*}$, a link that is $x+1$ away along the cycle is removed so that $s^{\prime}$ is minimal. As constructed, $\pi_{i}^{*}$ meets GCE conditions (ii) and (iii) for $i$, and given $\pi_{i}^{*}, s_{i}^{*}$ is a best response, thus meeting GCE condition (i) for $i$.

The key issue here is whether or not an individual can identify payoff-improving link initiations or removals. With "pessimistic" beliefs about the value of individuals in unseen parts of the network, an individual will not want to form new links, and those beliefs will not be contradicted by the signal. The key to the proof is establishing when an individual will not want to remove any links, i.e., when the signal that does not reveal any LLSs. Part (a) says that an equilibrium network cannot have cycles that are too small, but it can have cycles outside of players' observational ranges if, as shown in the proof, beliefs do not assign weight to states with cycles. Parts (b) and (c) concern an individual's ability to discern the existence of a LLS. Clearly, an LLS of size $y-1$ or smaller would be within $i$ 's observational range and thus could not exist in equilibrium. But GCE conditions (ii) and (iii) may also lead to beliefs that "identify" other LLSs even if they are not explicitly observed. It turns out that two conditions must be met to not be "identifiable": the player must have sufficient "unaccounted for utility" that could possibly (from the player's point of view) be attached to the LLS thus making the LLS not really a LLS, and there must be players whose values are not observed but who could be generating that unaccounted for utility. These conditions correspond to parts (b) and (c) of the Proposition.

Consider Fig. 2(c) for intuition. With $2 \in L L S_{1}^{2 / 1}$, are there beliefs $\pi_{1}$ such that keeping her link with 2 is a best response? For her current action to be a best response, there must exist at least one state $s^{\prime}$ given non-zero probability by $\pi_{i}^{*}$ in which the link with 2 is neither an LLS or part of a cycle (otherwise 1's best response must involve removing the link), and this $s^{\prime}$ must have some player $k$ or group of players $k_{1}, \ldots, k_{z}$, connected to 2 who makes 1 's link to 2 yield a marginal benefit greater than $c$ to player 1 . But this is not possible if $e$ is sufficiently small because there is only $\widetilde{v}_{1}^{2 / 1}(s, v)=2 e$ of unaccounted for utility. To meet GCE conditions (ii) and (iii), player 1 must have exactly $\widetilde{v}_{1}^{2 / 1}(s, v)=2 e$ of unaccounted for utility in any $s^{\prime}$ assigned non-zero probability, and even if all of that were gained solely through 1's link with 2 , that would still make the link with 2 worth only $3 e$ which is less than $c$ if $e$ is sufficiently small. Even though 1 cannot observe exactly who is connected to 2 , she would still recognize that the link to 2 is to a LLS because there is not enough received but unobserved utility that could make the link to 2 worthwhile. Thus, for any $\pi_{1}^{*}$ that meets GCE conditions (ii) and (iii), $s_{1}^{*}$ would not be a best response.

Whereas any (full information) NE network that is connected must also be minimal, ${ }^{9}$ such is not the case in a limited information GCE network. Proposition 1 establishes that the incorrect beliefs arising from limited information can lead to network outcomes in which there are too many links (cycles), too few links (disconnected or empty), or both (cycles in non-connected components). This difference arises due to the relaxing of the NE restriction that players' beliefs are correct in equilibrium. If players' have incorrect beliefs, then what they believe to be best responses might not in reality be best responses. Applying the GCE concept thus allows us to see how the incorrect beliefs resulting from limited information lead to outcomes different than those under full information. The lowest observation setting, $1 / 0$-link observation, has the largest set of equilibrium networks, ${ }^{10}$ and puts Proposition 1 in perspective.

Corollary 1. Fix $v$. $E_{1 / 0}(v)$ consists of all $s^{*}$ without direct redundant links such that $u_{i}\left(s^{*} \mid v\right) \geq v_{i}$ for all $i$.

\footnotetext{
${ }^{9}$ For a formal proposition and proof of this claim, see the technical appendix on the author's web site http://www.economics.uci.edu/ mcbride/.

${ }^{10}$ It can be shown that $E_{x / y} \supseteq E_{x^{\prime}} / y^{\prime}$ when $x \leq x^{\prime}$ or $y \leq y^{\prime}$ or both. See the technical appendix on the author's web site http://www.economics.uci.edu/ mcbride/.
} 
Proof. Under 1/0-link observation, condition (a) of Proposition 1 implies that $s^{*}$ can have any cycle with 3 or more players, so the only cycles not allowed are cycles of 2 players, which would be direct redundant links. Because $y=0$ implies $i$ observes only her own type, it follows that $L L S_{i}^{x / y}=I_{i}$, $\tilde{v}_{i}\left(s^{*}, v\right)=u_{i}\left(s^{*} \mid v\right)-\left(v_{i}-c\left|I_{i}\right|\right)$, and $v_{i j+}\left(s^{*}, v\right)=0$ for all $j \in L L S_{i}^{x / y}$ for each $i$. Condition (b) in Proposition 1 thus becomes $u_{i}\left(s^{*} \mid v\right)-\left(v_{i}-c\left|I_{i}\right|\right) \geq c\left|I_{i}\right| \Rightarrow u_{i}\left(s^{*} \mid v\right) \geq v_{i}$. According to condition (c) of Proposition 1 , any $s^{*}$ must have a player $y+1=1$ links away from $i$ along the path through $j \in I_{i}$. This is true in any network because $j$ herself is one link away from $i$.

The $u_{i}\left(s^{*} \mid v\right) \geq v_{i}$ condition in Corollary 1 is naturally interpreted as a participation constraint. In other words, any network without direct redundant links that makes each $i$ no worse off than being isolated can be an equilibrium under $1 / 0$-link observation. This includes the empty network, nonminimal and disconnected networks, or minimally connected networks. When $v_{i}>c$ for all $i$, every $s \in S$ without direct redundant links is in $E_{1 / 0}(v)$ because the participation constraint is met in any such $s$. That is, if all players are high-valued then literally any network (without directly redundant links) can be sustained as an equilibrium. This "anything goes" type result occurs because each i's observation is so limited that we can construct beliefs that make her believe her component is minimal and that any other player not in her component is isolated and low-valued.

\section{Incomplete information vs. imperfect monitoring}

To consider the different impacts of incomplete information and imperfect monitoring, compare the $\infty / 0$-link observation (perfect monitoring) setting where every $i$ perfectly monitors $s$ but does not observe any others' types with the $1 / \infty$-link observation (complete information) setting where every $i$ knows $v$ but only observes her own direct links. This second setting violates the $x \geq y$ assumption but helps us understand the different effects of the two types of limited information.

Perfect monitoring implies that any cycle would be identified, so any non-minimal structure could not be an equilibrium under perfect monitoring. The empty network (which is minimal) is clearly an equilibrium because each $i$ can have $\pi_{i}^{*}$ assign probability 1 to the empty network with $v_{j}=\bar{v}<c$ for all $j \neq i$. Moreover, a minimally connected network with the participation constraint met will also be an equilibrium because $\pi_{i}^{*}$ can assign probability 1 to a state where her link initiations yield a return higher than the link cost. Non-empty, disconnected networks that are minimal and meet the participation constraint are also equilibria. Even though $i$ observes some component $N_{j}, \pi_{i}^{*}$ may assign a low value to that component, thus preventing $i$ from wanting to initiate a link to players in the other components. Because she has no direct observation of the value of $N_{j}$, this belief meets GCE conditions (ii) and (iii). Of course, $i$ might believe that $j$ would only be in some component $N_{j} \neq N_{i}$ if her own participation constraint were met, thereby implying to $i$ that the value of $N_{j}$ exceeds $c$. Thus, if players attribute rationality to the other players, the disconnected, non-empty networks will not be equilibria, as $i$ will link to $j \in N_{j} \neq N_{i}$. Mutual, not common, knowledge of rationality is sufficient for this last result.

Proposition 2. Fix $v$. $E_{\infty / 0}(v)$ consists of all minimal $s^{*}$ (including the empty network) such that $u_{i}\left(s^{*} \mid v\right) \geq v_{i}$ for all $i$. With mutual knowledge of rationality, $E_{\infty / 0}(v)$ consists of the empty network and all minimally connected $s^{*}$ such that $u_{i}\left(s^{*} \mid v\right) \geq v_{i}$ for all $i$.

Under $1 / \infty$-link observation, the empty network is an equilibrium if $v_{i}<c$ for all $i$ but not when $v_{i}>c$ for at least one $i$. Any isolated player's beliefs must assign the high value to any highvalued player, so she could not be isolated in equilibrium. Making stronger claims about equilibrium networks will depend in a complicated way on a player's ability to discern, using her observation and knowledge of her utility, which players are in her component. Because of this, it is not possible to give precise necessary and sufficient conditions for connected equilibria for generic $v$. I instead examine two special cases.

The first scenario is one in which each $i$ can identify from her utility alone which others are in her component. Say that type profile $v$ is "distinct" if $\sum_{j \in N^{\prime}} v_{j} \neq \sum_{j \in N^{\prime \prime}} v_{j}$ for all subsets $N^{\prime}, N^{\prime \prime} \subseteq N$. In essence, each player's fact is sufficiently different from another so that the sum of any combination of players will differ from the sum of any other combination of players. One example of distinct types 
with $n=5$ is $v=\{1,10,100,1000,10000\}$. Notice that if numbers are picked at random from a continuous distribution, then any $v$ profile is generically distinct. The second scenario, studied by McBride (2006a) considers the symmetric types case with $v_{i}=\widehat{v}>c$ for all $i$.

Proposition 3. (a) Fix $v$, and suppose $v_{i}>c$ for at least one $i$. If $v$ is distinct, then any $s^{*} \in E_{1 / \infty}(v)$ must be connected with $u_{i}\left(s^{*} \mid v\right) \geq v_{i}$ for any $i$.

(b) [From McBride (2006a)] Fix $v$ with $v_{i}=\bar{v}>c$. (i) If $n<8$ then any $s^{*} \in E_{1 / \infty}$ is connected, but if $n \geq 8$ and c sufficiently close to $v$ then $E_{1 / \infty}$ also contains disconnected networks. (ii) With common knowledge of rationality, every $s^{*} \in E_{1 / \infty}$ is connected for any $n>1$.

If $v$ is distinct and known by each $i$, then any $\pi_{i}^{*}$ that meets GCE conditions (ii) and (iii) must identify the players in $i$ 's component. If $N_{i}$ does not include any $j$ with $v_{j}>c$ (or if $i$ is isolated) then $i$ 's current action cannot be a best response because according to $\pi_{i}^{*}$, initiating a link to that $j$ would make $i$ strictly better off. Of course, cycles outside of the observational range can still exist in equilibria. Thus, distinct types with at least one high-valued player is sufficient to have a connected network, but it cannot guarantee minimality. See McBride (2006a) for details and intuition of part (b).

Neither perfect monitoring nor complete information are sufficient alone for connectedness or minimality, however each does have a different impact on network efficiency. Perfect monitoring has an immediate implication about network architecture in that it implies minimality, but connectedness requires mutual knowledge of rationality because only then does it allows players to infer something about the value of other components. The impact of monitoring on connectedness is still limited, though, because the empty network is always an equilibrium even if all players are high-valued. Complete information of types $v$, unlike perfect monitoring, does not imply anything about minimality, but it does imply that any equilibrium is non-empty if at least one player is highvalued. Whether or not it guarantees a connected equilibrium will depend, first, on whether players can identify their component members and, second, on whether players ascribe rational behavior to other players. Thus, loosely speaking, complete information and perfect monitoring each provide one aspect of efficiency, but in general neither alone is sufficient for efficiency even when every Nash Equilibrium is efficient.

\section{Other considerations}

\subsection{Strict equilibria}

The strictness refinement changes " $\geq$ " to " $>$ " in condition (i) of the GCE definition. Researchers (Bala and Goyal, 2000, Galeotti et al., 2006) like this restriction because it greatly refines the set of network equilibria The restriction also has the nice dynamic property that strict equilibria are absorbing states in repeated game settings. According to a "link-switching" argument, strictness rules out as an equilibrium a situation in which a player's observation reveals that her utility is the same if she switches a link from $j$ to $k$. Bala and Goyal (2000) show that the center-sponsored star (wherein one $i$ initiates all links and no other player initiates links), is the only component architecture immune to this link-switching under full information. McBride (2006a) extends the result to the $x / \infty$-link observation case with $x \geq 2$. Intuitively, to know of the link-switching opportunity a player only needs observe a neighbor's link. I here extend this result the incomplete information case.

Proposition 4. Suppose $x \geq 2$. Then for any y under $x / y$-link observation, any strict GCE component must be a center-sponsored star.

Proof. Suppose equilibrium $s^{*}$ has component $N_{i}$ that is not a center-sponsored star. Thus, there must exist an $i, j \in N_{i}$ who both initiate links. If $\left|N_{i}\right|=2$ then it must be true that $s_{i j}^{*}=s_{j i}^{*}=1$, which is a direct redundant link that cannot exist in an equilibrium with $x \geq 1$.

Now suppose $\left|N_{i}\right|>2$. Again, there must be $i, j \in N_{i}$ who both initiate links. If $d(i, j)=1$ and, without loss of generality, $s_{i j}^{*}=1$, then $i$ observes $I_{j}$. If $s_{j i}^{*}=1$, then $i$ 's link is again observed to be redundant and cannot be an equilibrium. If $s_{j i}^{*}=0$ then there must be some $k$ such that $s_{j k}^{*}=1$. Since $i$ observes this link, $i$ observes that she receives no decrease in utility by setting $s_{i j}=0$ and $s_{i k}=1$, 
so $s^{*}$ is not a strict equilibrium. If $d(i, j)>1$, then there must be a player $k$ in the path between $i$ and $j$, and, without loss of generality, let $s_{i k}^{*}=1$. Now $i$ observes that she can switch her link from $k$ to any player directly connected to $k$ on the path to $j$ and receive the same utility, which implies $s^{*}$ is not a strict equilibrium.

A few comments deserve mention. First, center-sponsored stars are minimal, so in any strict equilibrium, each non-empty component is arranged efficiently even if the network as a whole is not efficient. Second, non-empty strict equilibria might not exist even when non-empty weak equilibria do. For example, if $v_{i}=c-\varepsilon, \varepsilon>0$ small for all $i \in N$, then a periphery-sponsored star (a star network in which the peripheral players all link to the central player, whom does not initiate any links) is a weak equilibrium (and efficient), but the only strict equilibrium is the empty network. Third, strict equilibria might not exist. If $v$ is distinct, has multiple high- and low-valued players, and is commonly known $(1 / \infty$-link observation), then any equilibrium must be connected. However, with $x \geq 2$, any strict equilibrium must also be a center-sponsored star, but such cannot be an equilibrium because an equilibrium cannot have a low-valued stem. Finally, strictness does not imply connectedness. In fact, with $x$ and $y$ finite, the empty network is always a strict equilibrium.

\subsection{Common prior}

Another way to refine the set of equilibria is to impose restrictions on players' beliefs, and a standard way to do this is to assume that individuals' types are drawn independently from a commonly known distribution $F_{v}$. Players' prior beliefs about others's types would equal this distribution, and players would, in equilibrium, Bayesian update given their signals. This approach gives individuals information they would not have without the assumption, and it will thus refine the set of equilibria. Consider $i$ who observes that $j$ is not in $N_{i}$. Without additional information to the contrary, $i$ must assign an expected value of linking to $j$ to equal the mean of $F_{v}$. If this expected value is greater than $c$, then $i$ has a profitable deviation (in expectation) to link to $j$. Thus, some disconnected networks that are GCE without assuming the common prior will no longer be equilibria. ${ }^{11}$

\subsection{Decay}

Flow decay captures the idea that the benefits of a link depreciate in the geodesic distance of that link. For example, information in a communication network is lost or altered as it passes through more people, so $i$ 's benefits from $j$ are higher if $j$ is closer to $i$ in the network. Not surprisingly, under full information flow decay often reduces the maximum distance between any $i$ and $j$ in the same component of an equilibrium because players want to be closer to one another to reduce decay. Sufficiently large decay ( $\delta$ close to 0 ) will mean that $i$ would rather form a direct link to $j$ than benefit from any indirect link to $j$ so that flow decay can lead to redundant links being efficient in equilibrium. This principle applies with incomplete information and imperfect monitoring, however, the implications of these ideas weaken as $x$ and $y$ decrease. In fact, flow decay has a somewhat minor effect when $x=1$ and $y=0$. It can be shown that with flow decay, the set of equilibrium networks under 1/0-link observation consists of all those that satisfy each player's participation constraint, akin to Corollary $1 .{ }^{12}$ The difference is that the decay reduces the overall value of most components, thus making fewer networks meet the participation constraint.

\subsection{Mutual consent networks}

Ties in many social networks requires the mutual consent and effort of both sides of the tie. McBride (2006b) examines the mutual consent setting under $x / y$-link observation and does so using

\footnotetext{
11 Proposition 4 holds in this common prior setting because the link switching has to do with observation of neighbors' links and not prior beliefs others' types.

${ }^{12}$ For a formal proposition and proof of this claim, see the technical appendix on the author's web site http://www.economics.uci.edu/ mcbride/.
} 
a new stability concept designed for the study of mutual consent network formation. He shows that the general impact of limited observation on network outcomes (i.e., expansion of the set of stable networks) applies in the mutual consent setting for the same reason it does here. However, he also shows that, unlike the model studied in this paper, many of the new equilibria that arise due to limited observation can actually be more efficient that the most efficient ones under full observation. The reason is that if individuals can maintain incorrect beliefs in equilibrium, then some of those beliefs may lead them to choose actions that are socially beneficial though individually detrimental. Whether there are unilateral link formation settings in which this result arises is a topic for future research.

\section{Conclusion}

This paper shows how and why imperfect monitoring and incomplete information lead to the existence of inefficient equilibria. Because empirical work suggests observation to be approximately $x=2$ and $y=2$ in many actual informal networks (Friedkin, 1983), my findings predict that many actual networks operate inefficiently. My findings also reveal that limiting players' information about others' ties has different implications than limiting information about players' types. Discovering how individuals act to lessen the network inefficiencies predicted by the above analysis constitutes a potentially fruitful avenue of research.

\section{Acknowledgement}

I thank Ben Polak, David Pearce, Stephen Morris, Amil Dasgupta, Sanjeev Goyal, Chris Udry, Dirk Bergeman, Venkatesh Bala, Hongbin Cai, Bill Zame, Matthew Jackson, Stergios Skaperdas, participants of the Topics in Game Theory group at Yale University, attendees at the 2003 South West Economic Theory (SWET) Conference and the 2007 Public Economic Theory Conference, UC Irvine colloquium participants, and anonymous referees for comments and support.

\section{References}

Bala, Venkatesh, Goyal, Sanjeev, 2000. A non-cooperative model of network formation. Econometrica 68 (5), 1181-1230.

Battigalli, Pierpaolo, Gilli, Mario, Molinari, M. Christina, 1992. Learning and convergence to equilibrium in repeated strategic interactions: An introductory survey. Ricerche Economiche 46 (3-4), 335-378.

Bondonio, Daniele, 1998. Predictors of accuracy in perceiving informal social networks. Social Networks 20 (4), 301-330.

Casciaro, Tiziana, 1998. Seeing things clearly: Social structure, personality, and accuracy in social network perception. Social Networks 20 (4), 331-351.

Dutta, Bhaskar, Jackson, Matthew (Eds.), 2003. Networks and Groups: Models of the Strategic Formation. Springer-Verlag, Heidelberg.

Friedkin, Noah E., 1983. Horizons of observability and limits of informal control in organizations. Social Forces 62 (1), 54-77.

Fudenburg, Drew, Levine, David, 1993. Self-confirming equilibrium. Econometrica 61 (3), 523-545.

Galeotti, Andrea, Goyal, Sanjeev, Kamphorst, Jurjen, 2006. Network formation with heterogeneous players. Games and Economic Behavior 54 (2), 353-372.

Gilli, Mario, 1999. On non-Nash equilibria. Games and Economic Behavior 27 (2), 184-203.

Kumbasar, Ece, Romney, A. Kimball, Batchelder, William, 1994. Systemic biases in social perception. American Journal of Sociology 100 (2), 477-505.

Laumann, Edward, 1969. Friends of urban men: An assessment of accuracy in reporting their socioeconomic attributes, moral choice, and attitude agreement. Sociometry 32 (1), 54-69.

McBride, Michael, 2006a. Imperfect monitoring in communication networks. Journal of Economic Theory 126 (1), 97-119.

McBride, Michael, 2006b. Limited observation in mutual consent networks. Advances in Theoretical Economics 6 (1), Article 3.

Rubinstein, Ariel, Wolinsky, Asher, 1994. Rationalizable conjectural equilibrium: Between nash and rationalizability. Games and Economic Behavior 6 (2), 299-311.

Stokman, Frans N., Doreian, Patrick, 1997. Evolution of social networks: Processes and principles. In: Doreian, P., Stokman, F.N. (Eds.), Evolution of Social Networks. Gordon and Breach Publishers, Amsterdam, pp. 233-250.

Wasserman, Stanley, Faust, Katherine, 1994. Social Network Analysis: Methods and Applications. Cambridge University Press, Cambridge. 